\title{
Assessment and treatment of breakthrough cancer pain: from theory to clinical practice
}

\author{
This article was published in the following Dove Press journal: \\ Journal of Pain Research \\ 12 September 2017 \\ Number of times this article has been viewed
}

\author{
Renato Vellucci' \\ Rocco Domenico Mediati' \\ Silvia Gasperoni' \\ Massimo Mammucari ${ }^{2}$ \\ Franco Marinangeli ${ }^{3}$ \\ Patrizia Romualdi ${ }^{4}$ \\ 'Palliative Care and Pain Therapy \\ Unit, University Hospital of Careggi, \\ Florence, ${ }^{2}$ Primary Care Unit, ASL \\ RM I, Rome, ${ }^{3}$ Department of Life, \\ Health and Environmental Sciences, \\ University of L'Aquila, l'Aquila, \\ ${ }^{4}$ Department of Pharmacy and \\ Biotechnology, University of Bologna, \\ Bologna, Italy
}

\section{Video abstract}

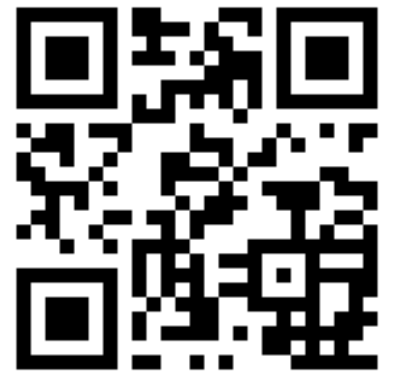

Point your SmartPhone at the code above. If you have a QR code reader the video abstract will appear. Or use: http://youtu.be//KgsXsvnuNwk

Correspondence: Renato Vellucci Palliative Care and Pain Therapy Unit, University Hospital Careggi Florence, Largo Brambilla N.3, 50I34 Florence, Italy

Tel +3955 7947734

Fax +39557947031

Email renato.vellucci@gmail.com
Abstract: Breakthrough cancer pain $(\mathrm{BTcP})$ is a common condition in oncological patients. However, its management is still suboptimal. Improved knowledge of BTcP and its management in clinical practice may have immediate importance for all physicians involved in the supportive care of cancer patients. This review critically discusses the most important concepts for the correct diagnosis of BTcP and presents some intriguing cases of the management of this condition in clinical practice. Overall, the most appropriate therapeutic choice appears to be a rapid-onset opioid (ROO), and in particular, the nasal route of administration is the quickest and most convenient mode of administration for the management of BTcP, especially when the patient needs rapid resolution of pain. To this end, intranasal fentanyl spray may have a particular relevance in clinical practice. Future research should focus on accepted definitions of BTcP to investigate the optimal management of this highly heterogeneous pain condition. Therapeutic decision-making of patients, clinicians, and payers will likely be driven from results of welldesigned clinical trials of ROOs.

Keywords: breakthrough cancer pain, rapid-onset opioid, intranasal fentanyl spray

\section{Introduction}

In oncological patients, breakthrough cancer pain (BTcP) is reported in $\sim 64.8 \%$ of subjects and is a major indicator of poor clinical outcome and lower efficacy of opioid therapy. ${ }^{1-3}$ Limited data are available on the Italian situation, but percentages between $40 \%$ and $50 \%$ have been reported. ${ }^{4,5}$ Moreover, patients suffering from BTcP present a decreased functional capacity and increased risk of depression and anxiety. BTcP represents a social cost as it affects productivity, and it also has a major impact on the patient's and caregivers' quality of life (QoL), heavily interfering with daily activities in $81 \%$ of patients. ${ }^{3}$

Poor BTcP management exposes the patient to a further worsening of conditions and also increases the costs of care for the health care system; however, multiple evidence suggests that BTcP is often managed suboptimally. ${ }^{6,7}$ An Italian study highlighted that about one-third of patients suffering from BTcP do not receive any kind of rescue therapy ${ }^{4}$ and another $33 \%$ of them received a World Health Organization (WHO) ladder level-one drug, with morphine more frequently administered than transmucosal fentanyl formulations. Similarly, disappointing data were reported in an European survey. ${ }^{3}$ Further evidence shows how in North America and Europe, BTcP is currently not optimally managed. ${ }^{7}$

Improved knowledge of BTcP and its management in clinical practice may have immediate importance for all physicians involved in the supportive care of cancer 
patients. This review critically discusses the most important concepts for the correct diagnosis of BTcP and presents some intriguing cases of the management of this condition in clinical practice.

\section{Definition of BTCP}

BTcP was first described by Portenoy in $1990 .{ }^{8}$ This initial definition has been extensively discussed. For instance, in 2002, BTcP was the focus of a semantic debate, leading to a consensus meeting of the expert working group of the European Association for Palliative Care. ${ }^{9}$ This group of experts introduced the terms "episodic" or "transient", both more straightforward than "breakthrough", a term that has no literary translation in several languages. Despite this effort, today the term "breakthrough pain" remains the only one used in medical literature. Indeed, the adoption of a terminology such as "episodic pain" increases the gap between experienced pain therapists and less experienced clinicians, thus introducing a further semantic complication.

Nowadays, the widely accepted definition of BTcP is "a transient exacerbation of pain that occurs either spontaneously or in relation to a specific predictable or unpredictable trigger despite relative stable and adequately controlled background pain". ${ }^{10}$ Usually, BTcP reaches its peak in severity within 5 minutes from onset and lasts up to 30-60 minutes. ${ }^{11,12}$ Recently, an Italian Expert group remarked that $\mathrm{BTcP}$ should be defined as a relevant change in pain intensity up to severe intensity in patients effectively treated with opioids, presumably at doses $\geq 60 \mathrm{mg}$ of oral morphine equivalents (able to provide analgesia to a mild pain intensity for most hours of the day). ${ }^{13}$ However, the discussion is still open, since in some cases, transient cancer pain exacerbations occur also without background pain, when background pain is uncontrolled, and regardless of opioid treatment. Last, it is important to remind that cancer pain exacerbations other than BTcP exist, that is the recently defined episodic pain. ${ }^{14}$

Diagnostic algorithms for assessment of BTcP are available (Figure 1). ${ }^{15}$ However, they show a variability of sensitivity and specificity when the cutoff that defines controlled background pain changes from mild-to-moderate and vice versa. Comprehensive clinical assessment remains the preferred method to approach BTcP. ${ }^{15}$

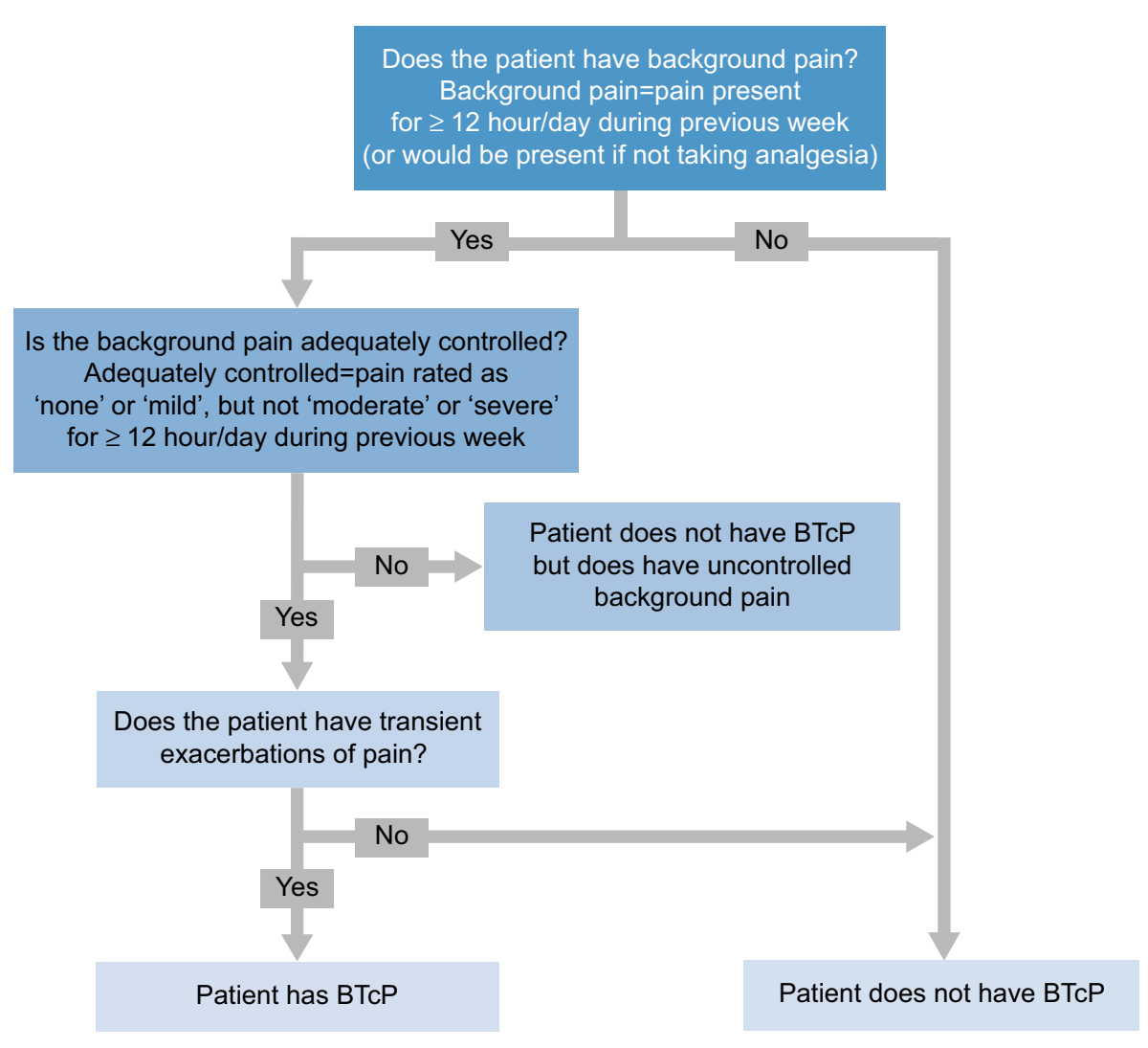

Figure I Diagnostic algorithms for assessment of BTcP.

Abbreviation: BTcP, breakthrough cancer pain. 


\section{Categorization of BTcP}

$\mathrm{BTcP}$ is variable, at both an inter-individual and even an intra-individual level. ${ }^{16}$ It can comprise different pathophysiological determinants (nociceptive, neuropathic, and mixed) and can be related to multiple causes (oncological disease, treatment, and concomitant diseases).

BTcP is usually divided into two subtypes:

- Incident pain, which is of predictable nature since it is precipitated by identifiable factors. Incident pain is further categorized into three subclasses:

o volitional incident pain, caused by a voluntary act (e.g., movement, dressing changes)

o non-volitional incident pain, caused by an involuntary act (e.g., bowel spasm)

o procedural pain, related to a diagnostic or therapeutic intervention (e.g., wound dressing)

- Spontaneous or idiopathic pain occurs in the absence of any specific activity and is therefore unpredictable.

Volitional and non-volitional incident pain was reported in up to $94 \%$ of cancer patients. ${ }^{17}$ In a recent Italian survey, the percentage of incident-type and idiopathic BTcP was $43.9 \%$ and $56.1 \%$, respectively. ${ }^{13}$ Incident pain is a predictor of poor success of pharmacological therapy. ${ }^{18}$ In an observational study on 1,000 $\mathrm{BTcP}$ patients, those with incident pain reported more interference with walking and working ability. ${ }^{3}$ It is worth noting that the inclusion of procedural $\mathrm{BTcP}$ precipitated by diagnostic and treatment procedures in this categorization remains controversial, mostly due to the possible absence of background therapy. With respect to idiopathic BTcP, a recent Italian survey reported that $56 \%$ of $\mathrm{BTcP}$ cases could be classified as spontaneous or idiopathic BTcP. ${ }^{13}$ Noteworthy, patients with spontaneous pain reported more interference with mood and sleep than those affected from other types of BTcP. ${ }^{3}$

In addition to the abovementioned entities, "pain from end dose" (or end-dose pain [EDP]) may be considered. It occurs just before the next scheduled dose of around-the-clock (ATC) analgesia, and it is attributable to an inadequate ATC analgesic dose or too prolonged interval between subsequent administrations.

In the next paragraphs, different cases explanatory of this classification are provided as an educational tool to facilitate understanding of the diagnostic and therapeutic processes. All patients provided written informed consent for the anonymized case details to be published, and the Tuscany Region Ethics Committee Area Vasta Centro Section approved the publication of those cases.

\section{Volitional incident pain}

In this case report, we are dealing with incidents episode of $\mathrm{BTcP}$ predictable because precipitated by volitional factors, specifically meals. The patient was a 65 -year-old woman suffering from pancreatic cancer and metastasis to lung and liver. She described her pain as stable and of mild intensity (numerical rating scales [NRSs], score of 2), with exacerbations only after food intake in the epigastric region (NRS 7), with irradiation to the back. The patient reported some episodes of nausea, vomiting, and oral mucositis grade 2 . She was treated with fentanyl patch $75 \mu \mathrm{g} / 72$ hours, methyl prednisolone $8 \mathrm{mg}$, and pantoprazole $40 \mathrm{mg}$. During the first visit at the palliative care unit, the treatment was modified introducing morphine immediate release (IR) $30 \mathrm{mg}$ 1 hour before any meal and she was referred to a nutritional specialist for nutritional plan. During the second visit, the patient reported morphine therapy as poorly effective and the concomitant onset of hallucinatory episodes and dizziness. Consequently, the patient was recommended to initiate titration with fentanyl nasal spray (INFS) $50 \mu \mathrm{g}$ administered 20 minutes before any meal. The patient identified $100 \mu \mathrm{g}$ as the dose with the best risk: benefit ratio and achieved a pain reduction of $60 \%$, reporting only sporadic need for a second puff at the end of the meal (20-30 minutes after the first intake).

\section{Non-volitional incident pain}

The patient was a 41-year-old man suffering from cholangiocarcinoma with liver metastasis. He described his pain as continuous as stable (NRS 1) in right upper quadrant of abdomen, with exacerbations precipitated by bowel gaseous distension (NRS 10). The colic episodes were recurring once per day on average, with severe intensity (NRS 10), sudden onset, and duration $<45$ minutes. Despite the low number of $\mathrm{BTcP}$ episodes, the nutrition capacity and QoL of the patient were seriously compromised.

Recurrence of episodes was worse with assumption of vegetables, fruits, and pasta. Family physician and oncologist scheduled a treatment with diet plan and fentanyl $50 \mu \mathrm{g} /$ hour every 72 hours, methylprednisolone $8 \mathrm{mg}$ once a day, and omeprazole $40 \mathrm{mg}$ and ibuprofen $600 \mathrm{mg}$ as needed. During the visit at the palliative care unit, the patient reported considerable discomfort caused by sporadic episodes of BTcP mainly at day-time. Titration with INFS was then recommended during the second visit: the patient identified the dose with the best risk:benefit ratio to be $200 \mu \mathrm{g}$, and this treatment was associated with a $50 \%$ reduction in pain intensity. During the third visit, the background pain was controlled, and fentanyl 
patch was thus reduced to $25 \mu \mathrm{g} /$ hour every 72 hours, in order to minimize the impact on bowel function. Three days later, the patient described an improvement of BTcP episodes intensity, and background pain remained under control.

\section{Clinical comment}

These two cases highlighted the efficacy of INFS. This formulation has the advantage of flexibility in use and fast onset of effect, thus allowing further assumptions 10 minutes after the first. In fact, oral IR formulations of opioids such as morphine or oxycodone typically have an extensive first-pass effect. Moreover, their hydrophilic nature slows the onset of analgesia (Table 1). ${ }^{31}$ These IR formulations are characterized by a poor correlation of their analgesic effect with the clinical features of a typical BTcP episode, ${ }^{18}$ particularly when it has a rapid onset and short duration. Thus, the slow onset of analgesia in 20-30 minutes and peak analgesia in 60-90 minutes results in delayed analgesic onset. Furthermore, the prolonged duration of their effect (3-6 hours) overlaps the ATC therapy, thus increasing the risk of adverse effects. However, nasal fentanyl provided faster onset of analgesia and attainment of clinically relevant pain relief. ${ }^{19}$ Noteworthy, rapid-onset opioids (ROOs) including fentanyl offer superior profile of pain relief compared with placebo within the first 30 minutes after administration, while oral morphine performs only slightly better than placebo (Figures 2 and 3). ${ }^{20,21,23}$

\section{Procedural pain}

We report the cases of two patients with procedural pain. The former patient was a 78-year-old man diagnosed with lung adenocarcinoma with multiple metastases to dorsal and lumbar spine. Pain symptoms were successfully treated (NRS 3) with oxycodone extended release (ER) $30 \mathrm{mg}$ per day in two administrations and methylprednisolone $8 \mathrm{mg}$, without any

Table I Characteristics of IR opioids

\begin{tabular}{|c|c|c|c|c|}
\hline Hydrophilic & IR opioid & $\begin{array}{l}\text { Onset of analgesia } \\
\text { (minutes) }\end{array}$ & $\begin{array}{l}\text { Duration of } \\
\text { effect (hours) }\end{array}$ & Advantages (A)/disadvantages (D) \\
\hline & \multirow[t]{2}{*}{ Morphine (oral) } & $30-40$ & 4 & A - available in multiple dosage forms, liquid concentrate \\
\hline & & & & D - slow onset of analgesia for idiopathic BTcP \\
\hline & Oxycodone (oral) & 30 & 4 & Same as morphine \\
\hline & Hydromorphone (oral) & 30 & 4 & $\begin{array}{l}\text { D - no liquid concentrate, slow onset of analgesia far idiopathic } \\
\text { BTCP }\end{array}$ \\
\hline & \multirow[t]{2}{*}{ Methadone (oral) } & $\sim 10-15$ & 4-6 & A - faster onset of analgesia in one small study \\
\hline & & & & D - complex pharmacology, pharmacokinetics \\
\hline & Fetanyl (transmucosal) & $\sim 5-10$ & $\mathrm{I}-2$ & A - fastest onset of analgesia \\
\hline Lipophilic & & & & $D$ - requires ongoing patient cooperation in use \\
\hline
\end{tabular}

Note: Copyright $\odot$ MediMedia. Reproduced with permission from Bennett D, Burton AW, Fishman S, et al. Consensus panel recommendations for the assessment and management of breakthrough pain: Part 2 management. $P$ and T. 2005;30(6):354-36I. ${ }^{31}$

Abbreviations: BTcP, breakthrough cancer pain; IR, immediate release.

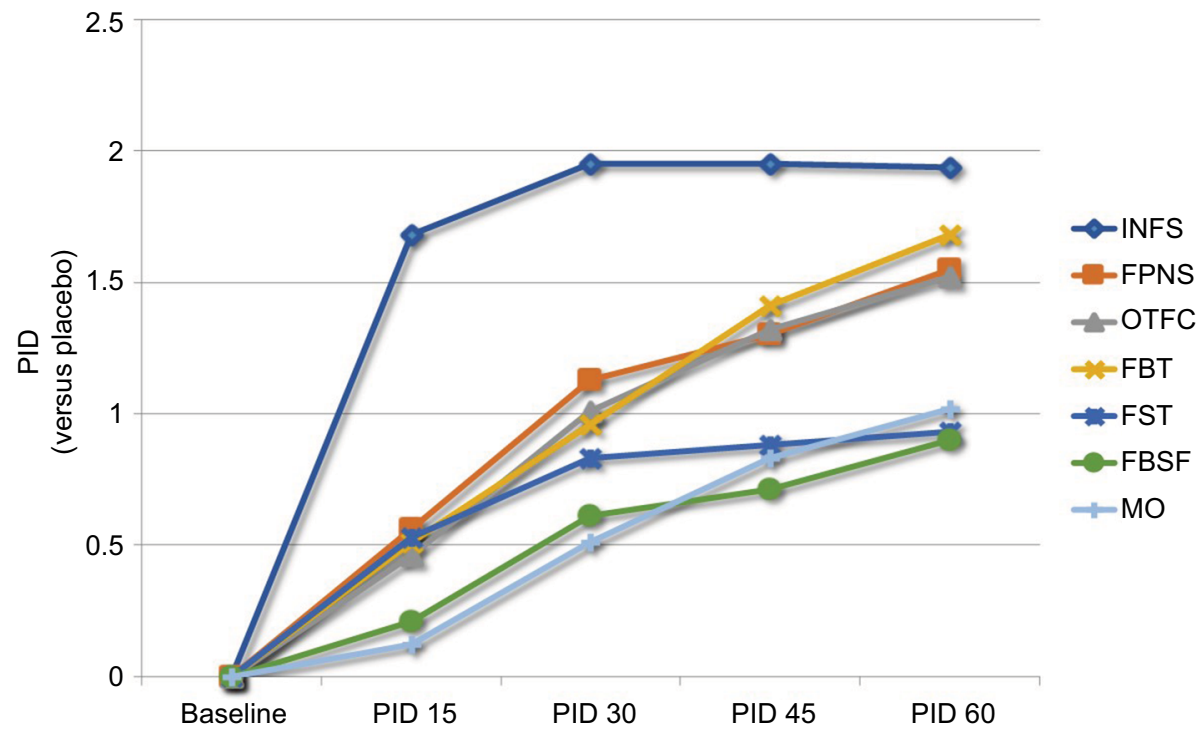

Figure 2 PID of BTcP medications relative to placebo.

Abbreviations: PID, pain intensity difference; BTcP, breakthrough cancer pain; INFS, intranasal fentanyl spray; FPNS, fentanyl pectin nasal spray; FST, fentanyl sublingual tablets; FBSF, fentanyl buccal soluble film; FBT, fentanyl buccal tablets; OTFC, oral transmucosal fentanyl citrate; MO, morphine sulfate immediate release. 


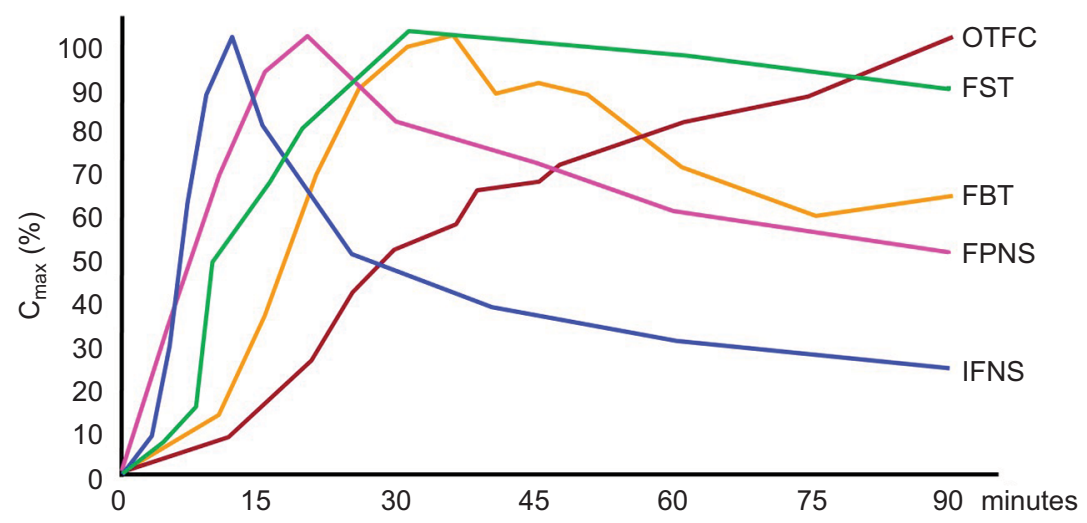

Figure 3 Pharmacokinetics of different formulation of ROOs.

Note: Data from Darwish et al, ${ }^{32}$ Elsner et al, ${ }^{33}$ Lyseng-Williamson et al, ${ }^{34}$ and Christrup et al. ${ }^{35}$

Abbreviations: INFS, intranasal fentanyl spray; FPNS, fentanyl pectin nasal spray; FST, fentanyl sublingual tablets; FBT, fentanyl buccal tablets; OTFC, oral transmucosal fentanyl citrate; ROOs, rapid-onset opioids; Cmax, maximum serum concentration.

episode of BTcP. A cycle of radiotherapy (RT) of the spine metastases was planned. At the start of treatment, the patient was asked to remain still on an uncomfortable X-ray table for treatment design and RT imaging. Patient reported a sudden onset of breakthrough pain (NRS 8). The procedure was immediately stopped, and patient was treated with INFS $50 \mu \mathrm{g}$ obtaining in 8 minutes a reduction of $50 \%$ in pain intensity, without leaving the X-ray table. This therapeutic strategy permitted to complete RT treatment with satisfaction of patient and sanitary staff.

The second patient was a 75-year-old man affected from colorectal cancer with metastases to first lumbar spine vertebra and lung. Pain symptoms were successfully treated (NRS 1/2) with morphine $80 \mathrm{mg}$ per day in two administrations. Patient reported a painful exacerbation in the evening when going to bed, with modest impact on sleep; he received lorazepam $2.5 \mathrm{mg}$ and ibuprofen $400 \mathrm{mg}$ tacked after dinner. During the course of the disease, the patient was scheduled for RT of lumbar tract. When patient underwent immobilization for RT procedures, he was unable to stand still for the onset of back pain radiating to lumbar area, abdomen, and legs. At a second attempt despite taking ibuprofen $600 \mathrm{mg} 1$ hour before the intervention and two vials of oral IR morphine $10 \mathrm{mg}$ at the start of the procedure, severe pain occurred again and RT was not possible. At the third attempt, the patient was advised to take a dose of ibuprofen $600 \mathrm{mg} 1$ hour before the procedure and a dose of INFS $200 \mu \mathrm{g} 20$ minutes before the starting of the centering and a second dose to the onset of pain. He was able to complete the procedure, remaining still for 50 minutes.

\section{Clinical comment}

These case reports deal with some common issues with RT, that is the onset of procedural pain due to the need to hold positions that cause pain in an isolated environment and the ethical factor related to their management. More than $50 \%$ of patients treated with RT experienced pain, and 39\% of them indicated that their pain was insufficiently treated. ${ }^{20,21}$ Noteworthy, pain caused by treatment and procedure was the most frequent type of pain experienced by oncological pediatric patients. $^{22}$

The onset of episodes of BTcP during RT design or delivery may delay or impede the completion of the procedure, increase costs, and may reduce the precision of RT. Furthermore, they can induce anxiety and can be associated with logistical issues due to RT rescheduling.

ROOs can be an option also in this case. In particular, INFS represents the fastest-based formulation of fentanyl available today with a favorable modality of titration, able to meet the needs of patients in this setting of care. ${ }^{23}$ In particular, before introducing an ROO, it is recommended that the patient is proved to be "opioid tolerant", taking at least $60 \mathrm{mg} /$ day oral morphine or an equi-analgesic dose of another oral opioid. Therefore, some authors recommend ROOs for managing induced procedural pain in patients already receiving an opioid for chronic pain. ${ }^{24}$ Despite the European Association for Palliative Care (EAPC) provided a weak recommendation for the administration of IR opioids to pre-emptively treat the predictable BTP episodes enough before the provoking maneuver, ${ }^{25}$ ROOs are characterized by improved pharmacokinetic profile than those formulations. Moreover, ROOs allow rapid titration, and this advantage can be particularly relevant for the treatment of procedural pain, which could be not completely predictable.

\section{Spontaneous or idiopathic pain}

The patient was a 59-year-old woman with adenoid cystic carcinoma of the left maxillary sinus and treated with subtotal maxillectomia. The patient reported stable mild pain (NRS 1), with exacerbations at the site of surgery (NRS 7) 
and irradiation to the temporal and periorbital regions. BTcP episodes recurred twice daily on average, in the form of electric shock and with severe intensity (NRS 10), sudden onset, and duration < 25 minutes. Pain was not associated with any trigger. The patient also presented stomatitis grade 2 and grade $2 / 3$ xerostomia.

Treatment with morphine $40 \mathrm{mg}$ BID, morphine IR $20 \mathrm{mg}$ on demand, dexamethasone $4 \mathrm{mg}$ once a day, pregabalin $150 \mathrm{mg}$ BID, and omeprazole $40 \mathrm{mg}$ was instituted. During the first visit at the pain therapy unit, the patient reported considerable discomfort and insomnia caused by sporadic episodes of BTcP, with major reduction of activities despite treatment with morphine on demand (defined as poorly effective). At the end of the visit, the patient was prescribed titration with INFS $50 \mu \mathrm{g}$ administered at the onset of the BTcP episode. The choice had fallen on nasal treatment given the problems of the oral cavity. In the subsequent visits, patient identified the $200 \mu \mathrm{g}$ dose as having the best risk:benefit ratio, and she achieved a $50 \%$ reduction of intensity of BTcP flares.

\section{Clinical comment}

The impossibility to identify any specific trigger or activities able to precipitate pain allows us to categorize this type of BTcP as spontaneous or idiopathic. This kind of BTcP is particularly difficult to treat because it can occur unpredictably, with little or no warning.

With respect to treatment of spontaneous BTcP, IR morphine sulfate is no more efficacious than a placebo within the first 45 minutes, and it is characterized by a pharmacokinetic profile that makes this formulation unsuitable for the majority of episodes (namely, rapid onset and short duration). ${ }^{17}$ In these patients, treatment with an ROO may be more effective than morphine administration given their faster onset of action.

\section{Pain from end dose}

The patient was a 71-year-old woman who was suffering from lung cancer with bone metastases (spine D12, L3, L2, and right ribs 2 and 3 ). Before the initiation of medical treatment, the oncologist started analgesic therapy with $20 \mathrm{mg}$ of oxycodone ER BID, ibuprofen $600 \mathrm{mg}$ BID, and omeprazole $40 \mathrm{mg}$. The patient reported continuous pain in the costal and lumbar area, of variable intensity (NRS 4-10). In the days, before the visit at the palliative care unit, the patient experienced painful episodes, mainly at evenings/nights. The first kind of episode had a slowly progressive onset in the lumbar area and occurred about 8/9 hours after regular dose of oxycodone. Moreover, episodes sporadically recurrent (once or twice per week), characterized by a rapid onset in the rib area, were reported, but they did not correlate with any identified cause. These flares had a fast onset, reaching a peak of intensity in 3-5 minutes, average duration of 20 minutes, intensity 9/10 NRS; in some cases, they disappeared without analgesics. In particular, nocturnal episodes were reported as particularly violent, probably also due to sleep deprivation.

The increase of the dosage of oxycodone to $40 \mathrm{mg}$ BID immediately resulted in the disappearance of recurrent episodes 8/9 hours after ATC analgesic intake. This allowed to classify this type of pain as EDP, while sporadic painful episodes were classified as spontaneous BTcP.

\section{Clinical comment}

EDP is sometimes considered a subtype of BTcP; it occurs just before a scheduled dose of analgesic. In this case, EDP was successfully managed by increasing the ATC dose of analgesics. However, in our opinion, since the definition of $\mathrm{BTcP}$ requires coexistence of a basic pain adequately controlled, ${ }^{3}$ the EDP is not classifiable as a subtype of BTcP.

\section{Critical issues of assessment and treatment of BTcP}

In addition to those highlighted in the above-described cases, several issues in the management of BTcP can be considered.

First, patients are the major source of information about the possible etiology, severity, and pattern of pain. ${ }^{26}$ Importantly, terms such as "BTcP" mean very little to most patients and relatives. The empowerment of patients and caregivers can promote and facilitate the assessment of BTcP (Table 2). ${ }^{5}$ Good communication skills can also aid the physician improve compliance and influence the patient's approach to opioid therapy. ${ }^{27}$ A successful treatment is closely linked to correct information about the safety profiles of the treatment options.

A second issue is to identify the most appropriate dosage of analgesic therapy and select the most suitable route of administration according to pain and patient characteristics. In this respect, sublingual and oral transmucosal routes are

Table 2 The process of empowerment

Trough deep information of patients and caregiver pursues multiple objectives

Understand the importance of BTcP and how to share clinical information about this condition

Learn about the different types of pain, with a special focus on BTCP Understand the terms used to describe different types of pain Learn to use a multidimensional pain day-book for the detection of pain Understand the peculiarities of analgesic drugs, with a special focus on ROOs

Abbreviations: BTcP, breakthrough cancer pain; ROOs, rapid-onset opioids. 
effective in reducing BTcP episodes. Nasal administration can take advantage of the favorable absorption and characteristics of the $150-180 \mathrm{~cm}^{2}$ of surface area of nasal mucosa. Drug administration through the nasal cavity allows a rapid absorption bypassing the oral route and offering a valid alternative also in the presence of oral mucositis, xerostomia, nausea, and vomiting - events frequently observed in cancer patients. Its high lipophilicity combined with short duration of action and low risk of irritation make fentanyl well suited for intranasal administration. Moreover, the nasal mucosal membrane is highly vascularized and directly in contact with the brain through the olfactory mucosa, making this route ideal for fentanyl absorption. INFS is one of two fentanyl nasal formulations approved for BTcP, and it is composed by an aqueousbuffered solution containing fentanyl at the dose of 50, 100, and $200 \mu \mathrm{g}$. The pharmacokinetic profile of INFS offers the highest bioavailability (89\%) among all the formulations available. A recent meta-analysis ${ }^{23}$ showed that INFS induces the greatest 15-minute post-baseline analgesic effect, producing an absolute pain intensity difference (PID) $\geq 2.5$, favoring INFS over other ROOs even at 30 minutes (Figure 4). Noteworthy, PID $\geq 2$ is considered clinically relevant in $\mathrm{BTcP}$ and represents an effective way to benchmark an analgesic medication. On these bases, INFS could be considered as the treatment of choice for patients suffering from episodes of BTcP characterized by fast onset and short duration (up to 1 hour). ${ }^{23}$ A preliminary longterm study in advanced cancer patients with BTcP has shown that INFS is well tolerated for prolonged periods of time and is effective in controlling episodes of BTcP. ${ }^{28}$

The third issue mainly concerns economic implications. Patients with BTcP are more likely to incur higher direct and indirect costs and may require additional health care resources

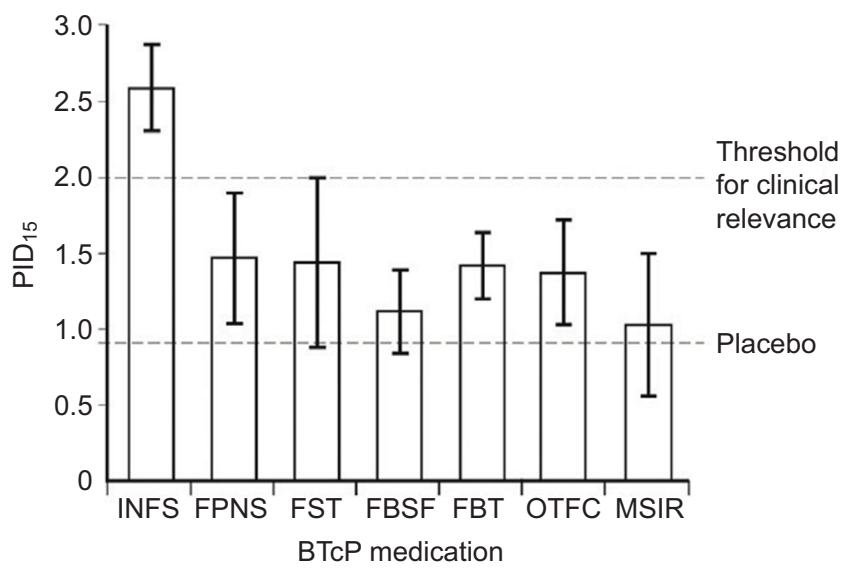

Figure 4 Estimate of absolute PID, 15 minutes after intake of BTcP medication. Abbreviations: BTcP, breakthrough cancer pain; INFS, initiate titration with fentanyl nasal spray; PID, pain intensity difference; FPNS, fentanyl pectin nasal spray; FST, fentanyl sublingual tablets; FBSF, fentanyl buccal soluble film; FBT, fentanyl buccal tablets; OTFC, oral transmucosal fentanyl citrate; MSIR, morphine sulfate immediate release. than patients without BTcP episodes, in particular more frequent medical and emergency visits and hospital admissions, with longer hospital stays. ${ }^{29}$ The correct utilization in clinical practice of ROOs reduces the burden of BTcP, and the analgesic efficacy of those drugs can be translated into savings for the National Healthcare System and improved QoL for patients. A recent cost-effectiveness analysis simulated, by a Markov model, the natural history of a hypothetical cohort of 100 advanced cancer individuals. ${ }^{30}$ The patients in the case arm received INFS, and those in the control arm received morphine. Treatment of BTcP with INFS costs 8,893 euros with an outcome of 0.63 quality-adjusted life years (QALYs), while the treatment with morphine costs 6,431 euros for a QALY of 0.29. These data generate an incremental cost-effectiveness ratio of 10,140 euros/QALY. Overall, the cost-effectiveness acceptability curve shows that the treatment of BTcP with INFS would have $86 \%$ provability of costing $<30,000$ euros/QALY, suggesting that this formulation is effective and sustainable.

\section{Conclusion}

BTcP may affect a large proportion of oncological patients, with major consequences on QoL. The most appropriate therapeutic choice appears to be an ROO, and in particular, the nasal route of administration is the quickest and most convenient mode of administration for the management of BTcP, especially when the patient needs rapid resolution of pain. Future research should focus on accepted definitions of BTcP to investigate the optimal management of this highly heterogeneous pain condition. Therapeutic decision-making of patients, clinicians, and payers will likely be driven from results of well-designed clinical trials of ROOs.

\section{Acknowledgments}

Editorial assistance for the preparation of this manuscript was conducted by Luca Giacomelli, PhD, and Aurora Mirabile, MD. This assistance was supported by internal funds.

\section{Disclosure}

Renato Vellucci received speaker/consultancy fees from Angelini, Grüenthal, Pfizer, Molteni, Norgine, Itafarmaco, TEVA and Sandoz. Rocco Domenico Mediati received speaker/consultancy fees from Angelini, Sigma-Tau, Pfizer, Molteni, Grüenthal, TEVA, and Mundipharma. Patrizia Romualdi received speaker/consultancy fees from Angelini, Molteni, Grüenthal and Sandoz. Massimo Mammucari received honoraria from Servier, Glaxo Wellcome, BioMedica, Pfizer, Merck Serono, Molteni, Serono Symposia International Foundation and Fondazione Lorenzini. The authors report no other conflicts of interest in this work. 


\section{References}

1. Bruera E, Fainsinger R, MacEachern T, Hanson J. The use of methylphenidate in patients with incident cancer pain receiving regular opiates. A preliminary report. Pain. 1992;50(1):75-77.

2. Caraceni A, Martini C, Zecca E, et al; Working Group of an IASP Task Force on Cancer Pain. Breakthrough pain characteristics and syndromes in patients with cancer pain. An international survey. Palliat Med. 2004;18(3):177-183.

3. Davies A, Buchanan A, Zeppetella G, et al. Breakthrough cancer pain: an observational study of 1000 European oncology patients. J Pain Symptom Manage. 2013;46(5):619-628.

4. Greco MT, Corli O, Montanari M, et al; Writing Protocol Committee; Cancer Pain Outcome Research Study Group (CPOR SG) Investigators. Epidemiology and pattern of care of breakthrough cancer pain in a longitudinal sample of cancer patients: results from the Cancer Pain Outcome Research Study Group. Clin J Pain. 2011;27(1):9-18.

5. Vellucci R. Breakthrough cancer pain. Eur J Oncol. 2015;20(suppl 1): 11-12.

6. Abernethy AP, Wheeler JL, Fortner BV. A health economic model of breakthrough pain. Am J Manag Care. 2008;14(5 suppl 1):S129-S140.

7. Bedard G, Davies A, McDonald R, et al. Breakthrough cancer pain: a comparison of surveys with European and Canadian patients. Support Care Cancer. 2015;23(3):791-796.

8. Portenoy RK, Hagen NA. Breakthrough pain: definition, prevalence and characteristics. Pain. 1990;41(3):273-281.

9. Mercadante S, Radbruch L, Caraceni A, et al; Steering Committee of the European Association for Palliative Care (EAPC) Research Network. Episodic (breakthrough) pain: consensus conference of an expert working group of the European Association for Palliative Care. Cancer. 2002;94(3):832-839.

10. Davies AN, Dickman A, Reid C, Stevens AM, Zeppetella G; Science Committee of the Association for Palliative Medicine of Great Britain and Ireland. The management of cancer-related breakthrough pain: recommendations of a task group of the Science Committee of the Association for Palliative Medicine of Great Britain and Ireland. Eur J Pain. 2009;13(4):331-338.

11. Davies A, Zeppetella G, Andersen S, et al. Multi-centre European study of breakthrough cancer pain: pain characteristics and patient perceptions of current and potential management strategies. Eur J Pain. 2011;15(7):756-763.

12. Zeppetella G. Breakthrough pain in cancer patients. Clin Oncol. 2011;23(6):393-398.

13. Mercadante S, Lazzari M, Reale C, et al; IOPS Study Group. Italian Oncological Pain Survey (IOPS): a multicentre Italian study of breakthrough pain performed in different settings. Clin J Pain. 2015;31(3):214-221.

14. Løhre ET, Klepstad P, Bennett MI, et al. European Association for Palliative Care Research Network. From "breakthrough" to "episodic" cancer pain? A European Association for Palliative Care Research Network Expert Delphi Survey toward a common terminology and classification of transient cancer pain exacerbations. J Pain Symptom Manage. 2016;51(6):1013-1019.

15. Webber K, Davies AN, Cowie MR. Accuracy of a diagnostic algorithm to diagnose breakthrough cancer pain as compared with clinical assessment. J Pain Symptom Manage. 2015;50(4):495-500.

16. Perry GF. The Diagnosis and Treatment of Breakthrough Pain. New York: Oxford American Pain Library; 2008.

17. Zeppetella G, Ribeiro MD. Pharmacotherapy of cancer-related episodic pain. Expert Opin Pharmacother. 2003;4(4):493-502.
18. Mercadante S, Maddaloni S, Roccella S, Salvaggio L. Predictive factors in advanced cancer pain treated only by analgesics. Pain. 1992;50(2):151-155.

19. Zeppetella G. Evidence-based treatment of cancer-related break through pain with opioids. J Natl Compr Canc Netw. 2013;11(suppl 1): S37-S43.

20. Janjan N. Do we need to improve pain management in the radiation oncology department? Nat Clin Pract Oncol. 2005;2:130-131.

21. Pignon T, Fernandez L, Ayasso S, Durand M, Badinand D, Cowen D. Impact of radiation oncology practice on pain: a cross-sectional survey. Int J Radiat Oncol Biol Phys. 2004;60(4):1204-1210.

22. Miser AW, Dothage JA, Wesley RA, Miser JS. The prevalence of pain in a pediatric and young adult cancer population. Pain. 1987;29(1):73-83.

23. Zeppetella G, Davies A, Eijgelshoven I, Jansen JP. A network metaanalysis of the efficacy of opioid analgesics for the management of breakthrough cancer pain episodes. J Pain Symptom Manage. 2014;47(4): 772.e5-785.e5

24. Poulain P, Michenot N, Ammar D, et al. Mise au point sur l'utilisation du fentanyl transmuqueux chez le patient présentant des douleurs d'origine cancéreuse. Douleurs. 2012;13:34-39.

25. Caraceni A, Hanks G, Kaasa S, et al; European Palliative Care Research Collaborative (EPCRC); European Association for Palliative Care (EAPC). Use of opioid analgesics in the treatment of cancer pain: evidence-based recommendations from the EAPC. Lancet Oncol. 2012;13(2):58-68.

26. Abrahm JL. Assessing the patient in pain. In: Abrahm JL, editor. A Physician's Guide to Pain and Symptom Management in Cancer Patients. 2nd ed. Baltimore, MD: Johns Hopkins University Press; 2005:107-147.

27. Rhiner MI, Von Gunten CF. Cancer breakthrough pain in the presence of cancer-related chronic pain: fact versus perceptions of health-care providers and patients. J Support Oncol. 2010;8(6):232-238.

28. Mercadante S, Vellucci R, Cuomo A, et al. Long-term efficacy and tolerability of intranasal fentanyl in the treatment of breakthrough cancer pain. Support Care Cancer. 2015;23(5):1349-1354.

29. Vellucci R, Mediati RD, Gasperoni S, et al. Pharmacoeconomic considerations about breakthrough cancer pain. Value Health. 2015;18(7): A665-A666.

30. Ruggeri M, Turriziani A, Oradei M. Cost-effectiveness analysis of transnasal fentanyl citrate for the treatment of breakthrough cancer pain. Expert Rev Pharmacoecon Outcomes Res. 2014;14(3): 459-464.

31. Bennett D, Burton AW, Fishman S, et al. Consensus panel recommendations for the assessment and management of breakthrough pain: Part 2 management. $P$ and T. 2005;30(6):354-361.

32. Darwish M, Kirby M, Robertson P Jr, Hellriegel E, Jiang JG. Comparison of equivalent doses of fentanyl buccal tablets and arteriovenous differences in fentanyl pharmacokinetics. Clin Pharmacokinetics. 2006;45(8): 843-850.

33. Elsner F, Zeppetella G, Porta-Sales J, Tagarro I. Newer generation fentanyl transmucosal products for breakthrough pain in opioid-tolerant cancer patients. Clin Drug Investig. 2011;31(9):605-618.

34. Lyseng-Williamson KA. Fentanyl pectin nasal spray: in breakthrough pain in opioid-tolerant adults with cancer. CNS Drugs. 2011;25(6): 511-522.

35. Christrup LL, Foster D, Popper LD, Troen T, Upton R. Pharmacokinetics, efficacy, and tolerability of fentanyl following intranasal versus intravenous administration in adults undergoing third-molar extraction: a randomized, double-blind, double-dummy, two-way, crossover study. Clin Ther. 2008;30(3):469-481. 
The Journal of Pain Research is an international, peer reviewed, open access, online journal that welcomes laboratory and clinical findings in the fields of pain research and the prevention and management of pain. Original research, reviews, symposium reports, hypothesis formation and commentaries are all considered for publication
The manuscript management system is completely online and includes a very quick and fair peer-review system, which is all easy to use. Visit http://www.dovepress.com/testimonials.php to read real quotes from published authors. 